\title{
RELATOS DE PESQUISAS
EMPLEO DE INSTRUMENTOS DE GESTIÓN DEL
CONOCIMIENTO PARA COMPRENSIÓN DE LOS PATRONES
DE PREFERENCIA DEL CONSUMIDOR EN PRODUCTOS DE LA CADENA DE LA MODA
}

\author{
Antonio Carlos Zambon \\ Doutor em Engenharia de Produção pela Universidade Federal de São Carlos, \\ Brasil. Professor da Universidade Estadual de Campinas, Brasil. \\ E-mail: zambon@ft.unicamp.br \\ Gisele Busichia Baioco \\ Doutora em Ciência da Computação e Matemática Computacional pela \\ Universidade de São Paulo, Brasil. \\ Professora da Universidade Estadual de Campinas, Brasil. \\ E-mail: gisele@ft.unicamp.br \\ Luz Amparo Torres Carreño \\ Mestrando em Tecnologia pela Universidade Estadual de Campinas, Brasil. \\ E-mail: luzatc93@gmail.com \\ Juan Fernando Galindo Jaramillo \\ Mestrando em Tecnologia pela Universidade Estadual de Campinas, Brasil. \\ E-mail: juan.jaramillo@pos.ft.unicamp.br
}

\begin{abstract}
Resumen
Para reducir riesgos de pérdida, aumentar la eficiencia en la producción y mejorar las ventas, las empresas deben conocer los deseos y necesidades de los consumidores. En pequeñas y medianas empresas, esta situación se dificulta por la falta de información que permita conocer mejor a los consumidores. El objetivo del presente trabajo fue analizar la estructura del modelo de preferencia del consumidor en productos de la moda, utilizando un método de adquisición y representación del conocimiento. La metodología llevada a cabo para atender este objetivo comenzó con el desarrollo de una visión estructurada sobre el modelo de preferencia del consumidor partiendo de la literatura sobre los factores que influyen tal preferencia. Para la extracción y representación del conocimiento fue utilizado el software BLUE KMS ${ }^{\circledR}$ que posibilitó la construcción de un mapa conceptual expandido que describe el modelo de preferencia del consumidor en productos de la moda. Este análisis permitió crear y simular un modelo apoyado en la estrategia de redes fast fashion para la producción, identificando las características principales de la estrategia y adaptando ese modelo a las realidades de las pequeñas y medianas empresas. El modelo de la estrategia fast fashion fue diseñado usando dinámica de sistemas a través del software STELLA 9.1.3. Por medio del mapa conceptual fue posible identificar los modelos mentales utilizados por los consumidores para elegir sus compras. Además, la simulación en dinámica de sistemas permitió entender el funcionamiento de la estrategia fast fashion y cómo ella podría ser adecuada en las empresas.
\end{abstract}

Palavras-chave: Moda rápida, Dinámica de sistemas, Mapa conceptual expandido, Pequeñas y medianas empresas, Inteligencia de mercado.

Perspectivas em Gestão \& Conhecimento, João Pessoa, v. 7, Número Especial, p. 50-65, mar. 2017. http://periodicos.ufpb.br/ojs2/index.php/pgc. ISSN: 2236-417X. Publicação sob Licença (cc)) EY-NC-ND. 


\title{
KNOWLEDGE MANAGEMENT TOOLS FOR PATTERN RECOGNITION OF CONSUMER PREFERENCE IN FASHION PRODUCTS
}

\begin{abstract}
In order to reduce the risks of products' loss, to raise efficiency in production, and to improve sales, enterprises must know the consumers' wishes and needs. In small and medium enterprises, this situation worsens, due to the lack of information about their customers. This paper's goal was to analyze the structure of consumer's preference model about fashion products, by using a knowledge acquisition and representation method. The methodology performed to achieve this goal started with the development of a structured vision on the consumer's preference model from literature about factors that influence consumers' preferences, in order to use this vision in small and medium enterprises, supporting internal decisions on production. For knowledge extraction and representation, we used BLUE KMS ${ }^{\circledR}$ software, which allowed the construction of an expanded concept map. This map described the consumer's preference model in fashion products. This analysis allowed to create and simulate a model supported by fast fashion strategies for production, identifying the main characteristics of these strategies, and adapting this model to small and medium enterprises' reality. The fast fashion modelling was designed through system dynamics by using STELLA 9.1.3 software. The concept map allowed the identification of the mental models used by consumers to purchase products. Besides, the system dynamics simulation allowed to understand the functioning of the fast fashion strategies and how they could be adapted to small and medium enterprises.
\end{abstract}

Keywords: Fast fashion, System dynamics, Concept map, Small and medium enterprises, Market intelligence.

\section{INTRODUCCIÓN}

Los métodos de producción utilizados en las organizaciones industriales competitivas permiten una sustitución constante de bienes según la dinámica del mercado. En una visión integral, los bienes durables son producidos con una frecuencia de cambios diferente a la de los bienes de consumo. En el ámbito de los diversos nichos de mercado, las características con que ocurren estos cambios es todavía más específica. En el sector de la moda, por ejemplo, existe una interdependencia entre los productos que comparten conceptos de preferencia que se sobreponen. Por esta razón, se hace necesario el estudio de toda la cadena de producción. La importancia de la cadena de moda brasileña tiende a proyectar al país como global fashion trendsetter (marcador global de tendencias de moda), poniéndolo a la par de los principales productores de moda, como Italia, Francia y Estados Unidos, en 2020 (AGIS et al., 2010). Esta cadena está formada por empresas de vestuario, calzado y accesorios, entre otras. Sus productos, pudiendo ser inclusive de segmentos industriales distintos, son interdependientes, pues están sometidos a un patrón de preferencia armónico, siendo que un producto puede ser preferido o descartado en función de otro. Por ejemplo, la elección de un calzado específico puede estar en función de otra pieza de vestuario, como un pantalón, y ambos pueden establecer un patrón de preferencia para un accesorio, como una pulsera.

De esta manera, se observa que la reorganización de un componente del vestuario puede representar un cambio en el patrón de elección global, aumentando la complejidad en la interpretación de las preferencias. Este reconocimiento puede provocar modificaciones en los patrones de ventas de toda la cadena. La globalidad en el patrón de elección que se observa diseminada en la cadena solo puede ser interpretada de forma sistémica, dada su abstracción y su importancia para la composición de las estrategias de oferta (SENGE, 1997). Así, las grandes estructuras minoristas del patrón Fast fashion (moda rápida) o Low cost (bajo costo) pueden interactuar fácilmente con esa globalidad, pues están presentes en toda la cadena de producción y distribución, teniendo acceso total al conocimiento distribuido en la cadena. En contraste, eso no ocurre con las micro, pequeñas y medianas empresas (PYME). Las PYME cumplen un papel importante en las economías locales y regionales, siendo que en 2013 , las aproximadamente 600.000

Perspectivas em Gestão \& Conhecimento, João Pessoa, v. 7, Número Especial, p. 50-65, mar. 2017. 
empresas pequeñas y medianas brasileñas representaban el $30 \%$ del producto interno bruto (PIB)(PRICEWATERHOUSECOOPERS, 2013). La cantidad de PYME existentes en la cadena de la moda es bastante significativa. En el sector textil-confección por ejemplo, entre 2007 y 2011, las empresas de porte medio representaron el $27 \%$ del total de las PYME y un 69\% de las PYME intensivas en mano de obra (UNIETHOS, 2013).

Con el fin de facilitar el análisis del conocimiento distribuido en la cadena de la moda, a partir de 2008, el Ministerio de Desarrollo, Industria y Comercio de Brasil (MDIC) lanzó, por medio de un programa gubernamental llamado Brasil Maior (Brasil Mayor), el proyecto Sistema de Moda Brasil (SMB). El SMB es un instrumento de política industrial que busca el desarrollo de los aglomerados productivos relacionados con la moda, siendo uno de sus objetivos la promoción de forma integrada de la moda brasileña en los mercados interno y externo. Según el MDIC, el proyecto se enfoca en un componente transversal a las cadenas asociadas a la moda que debe estar presente en sus productos. Tal componente es el "desarrollo de identidad a partir de la diversidad cultural brasileña como factor de adición de valor a los productos" (MDIC, 2015, traducción nuestra).

Para que puedan hacer parte de ese universo, las PYME brasileñas necesitan una estrategia adecuada que presupone tornar explícito el conocimiento tácito sobre la formación de un concepto común y eficiente de diferenciación, aprovechando la estructura de clústeres empresariales y el concepto de patrón de referencia o patrón global de elección.

Entretanto, lo que se verifica en el ambiente productivo, sobre todo en aquel formado por grupos de pequeñas y medianas empresas, es que persisten modelos mentales particionados con los cuales se tratan endógenamente los componentes que deberían ser compartidos en cadena. Por lo tanto, el patrón de preferencia del consumidor permanece desconocido y no es considerado en el desarrollo de los productos. Inclusive en Arreglos Productivos Locales apoyados por fomento gubernamental, se observa la prevalencia del modelo de ensayo y error en el desarrollo de los productos. A esto se suma la rivalidad entre las empresas del clúster por la disputa de los profesionales de diseño y por los proveedores de partes supuestamente diferenciadas y, en última instancia, por la clonación de productos exitosos en ventas.

Bajo este patrón de comportamiento, las pequeñas empresas, aisladas en sus modelos mentales partidos, apenas podrán competir si tuvieren acceso al conocimiento existente en los modelos capaces de representar los patrones de preferencia de productos de su segmento de mercado que permitan proyectar sus productos.

Partiendo de lo anterior, fue desarrollado un modelo simulado que considera las principales variables que constituyen los patrones de preferencia del consumidor (CHURCHILL; PETER, 2000). Tales variables fueron sometidas a los ambientes que establecen la dinámica del mercado (NENNI; GIUSTINIANO; PIROLO, 2013). Este esfuerzo busca reducir la falta de conocimiento que aflige a las PYME de la cadena de la moda, permitiendo que tomen decisiones con respecto a la producción. En el modelaje fue empleado un Mapa Conceptual Extendido - MCE (ZAMBON et al., 2016a), obteniendo un patrón representativo de la formación de preferencias de los consumidores. Para analizar la dinámica y la comprensión del contexto que define las preferencias, el MCE fue sometido a un simulador basado en Dinámica de Sistemas (DS) (ISEESYSTEMS, 2009). Mediante la simulación fue posible evaluar como la estrategia utilizada por las grandes redes fast fashion puede ser adaptada para generar resultados en las pequeñas empresas.

De este modo fue posible atender el objetivo de este trabajo, que fue analizar la estructura del modelo de preferencia del consumidor por medio de herramientas de Gestión del Conocimiento (GC). A través de los MCE revelamos los modelos mentales utilizados por consumidores para escoger sus compras. Además, la simulación por DS permitió entender la dinámica del empleo de tales modelos. Habiendo comprendido la lógica del modelo de preferencia del consumidor se hace posible la concepción de instrumentos de análisis de tendencias de mercado más ajustados, que permitirán a los pequeños emprendedores tomar decisiones sobre qué producir, cuánto producir y cómo producir.

Perspectivas em Gestão \& Conhecimento, João Pessoa, v. 7, Número Especial, p. 50-65, mar. 2017. 
El resto de este trabajo está organizado de la siguiente manera: Las secciones 2 y 3 presentan el referencial teórico del artículo: en la Sección 2 describimos algunos trabajos relacionados con la problemática en cuestión; por su parte, en la Sección 3, discutimos el modelo de preferencia del consumidor. En la Sección 4 abordamos los métodos de GC usados para modelar la preferencia del consumidor, específicamente MCE y DS. En la sección 5 se encuentran el modelaje resultante de la preferencia del consumidor y la simulación del fast fashion. Finalmente, en la Sección 6 mostramos las conclusiones sobre el análisis realizado.

\section{TRABAJOS RELACIONADOS}

En la literatura sobre identificación de patrones de preferencia del consumidor se encuentran trabajos que evalúan la influencia de un pequeño conjunto de variables en la preferencia del consumidor y trabajos que definen de forma general procesos de decisión de compra. Estos estudios son realizados con base en respuestas a estímulos identificados a través de recolección de datos vía web y respuestas a cuestionarios. En términos generales se observa que existe una tendencia por parte de los consumidores a escoger un determinado producto con base en factores como: temporada, clima, edad, economía, género, estilo de vida, intereses, marcas, entre otros. Sin embargo, no se encontraron estudios que identifiquen la interacción entre estas variables ni su influencia real en el proceso de elección del consumidor en el sector de la moda. A continuación se describen algunos trabajos representativos de la literatura que tuvieron como objetivo determinar factores que afectan la preferencia y la toma de decisiones del consumidor en el proceso de selección de un producto en el mercado de la moda.

CHEGINI, MOLAN y KASHANIFAR (2016) evaluaron el impacto de los valores culturales en la preferencia del consumidor en el mercado de la moda a través de una serie de cuestionarios de consumidores de marcas como Adidas, Nike y Holiday en la ciudad de Teherán. Los cuestionarios fueron clasificados en tres secciones: la primera sección obtiene información sobre datos demográficos, la segunda sección evalúa los valores culturales de los encuestados y la tercera sección permite la identificación de la personalidad de las marcas. Los resultados del estudio mostraron que la cultura es uno de los principales factores que influyen en el proceso de preferencia del consumidor, ya que las personas tienden a elegir las marcas que tienen características más cercanas a su personalidad.

ISMAIL, MASOOD y TAWAB (2012) determinaron factores que afectan la preferencia del consumidor sobre las marcas locales y marcas internacionales. Para esto, se realizaron cuestionarios a jóvenes en centros comerciales en Karachi, Paquistán. Los resultados mostraron que los factores más influyentes en el proceso de toma de decisión sobre un producto fueron el precio y la calidad del producto, dado que los consumidores asocian generalmente el precio de la marca con su calidad; otros factores influyentes fueron el etnocentrismo del consumidor, el país de origen de la marca, la condición social del consumidor, relatividad de precios con las marcas de la competencia, familia y amigos.

HALUK KÖKSAL (2007) determinó factores que afectan la preferencia del consumidor en el mercado de ropa infantil de Turquía mediante cuestionarios para padres de familia. Las respuestas a esos cuestionarios permitieron encontrar relaciones entre los hábitos de compra y las características demográficos de los encuestados. También definieron que los factores más importantes para la toma de decisiones de compra de ropa infantil son: la talla de la ropa, el precio, la calidad, la facilidad de compra y las opciones de pago. Factores como la moda, la marca y la difusión en internet no fueron importantes en la decisión de compra.

ALVEREZ, FAVERO y FRANCISCO (2013) identificaron la influencia de la publicidad sobre vestuario de moda en los consumidores, teniendo en cuenta variables como recordación, logo y mensaje de la marca. Para el estudio se realizaron cuestionarios estructurados en Brasil. Sus resultados mostraron que nombres cortos y logos simples con colores fuertes son más recordados

Perspectivas em Gestão \& Conhecimento, João Pessoa, v. 7, Número Especial, p. 50-65, mar. 2017. 
por los consumidores; publicidad que evidencie valores, comportamientos, experiencias y no el producto por sí solo atraen más a los consumidores. Este estudio permite identificar cómo el marketing juega un rol importante en el proceso de decisión sobre un producto y cuáles son los factores más relevantes a la hora de atraer la atención del consumidor.

KARIMI, PAPAMICHAIL y HOLLAND (2015) encontraron una tipología de la decisión de compra de los consumidores online basada en el estilo de toma de decisión del consumidor y su conocimiento sobre el producto a través de un análisis de videos que registraron los comportamientos de diferentes consumidores con relación a determinados productos en el Reino Unido. Los autores caracterizaron dos tipos de consumidor: el primer tipo basa su decisión de compra en que el producto cumpla con sus expectativas; el segundo decide luego de examinar todos los productos disponibles. Ese estudio encontró que un mayor conocimiento sobre el producto lleva a los consumidores del primer tipo a una toma de decisión rápida y un bajo conocimiento sobre el producto hace que los consumidores del segundo tipo tomen más tiempo en la toma de decisión.

Los trabajos encontrados en la revisión de la literatura permiten identificar los diferentes factores que influyen en el proceso de elección de un producto del sector de la moda. Con base en los factores identificados realizamos un estudio para determinar cómo tales factores se relacionan entre sí y así poder modelar los patrones de preferencia del consumidor.

\section{PREFERENCIA DEL CONSUMIDOR}

El fordismo y su línea de producción en masa buscaban atender el alto crecimiento del consumo en la industria automovilística en la década de 1920. El auge en las ventas de Ford fue ocasionada por productos con precios competitivos obtenidos con el aumento de la productividad alcanzada por la división del trabajo y la especialización. Sin embargo, la evolución de los patrones de oferta propuestos por el toyotismo con la producción en pequeños lotes, convirtió a la calidad del producto en la mayor preocupación para la industria y aumentó la variedad de modelos, conceptos, colores, etc. Así, Toyota abrió una nueva etapa de la producción en masa, teniendo en cuenta el enfoque en la satisfacción del cliente y reduciendo inventarios con la estrategia just-in-time (WOMACK; JONES; ROOS, 2007).

La satisfacción del consumidor, en el sentido moderno, está relacionada con el concepto económico de utilidad, que bajo una visión clásica es considerada sólo como una proporción directa de la cantidad, es decir, cuanto mayor es la cantidad de bienes disponibles, mayor será la satisfacción del consumidor. Esta lectura meramente cuantitativa fue modificada en el enfoque moderno en donde la utilidad trasciende la necesidad, representando también la medida de la satisfacción de las necesidades y deseos del consumidor (ELLSBERG, 1954).

Se entiende que la concepción de utilidad depende de la percepción individual y que el individuo utiliza su racionalidad para comparar productos, juzgando racionalmente la adquisición de uno u otro. Así, los consumidores con alto potencial de elección, teniendo en cuenta un paradigma de utilidad fuertemente apoyado en la satisfacción y el aumento de los ingresos, estarán probablemente inclinados a un modelo dinámico, cuya tasa de cambio estará asociada a la capacidad del mercado de ofrecer cada vez más productos diferentes.

El avance de la tecnología en procesos productivos garantiza la evolución en la diferenciación de productos, permitiendo a las industrias explorar la variedad y la calidad, aprovechando nuevas características como factores ganadores de ventas (PORTER, 1986). De esta manera, asociando la vanguardia de la producción a la flexibilidad, es posible mejorar las ventas apoyándose en la nosaciedad de consumo y en el concepto de satisfacción.

Varios sectores económicos han demostrado gran capacidad en la exploración de estas nuevas características del mercado, acortando el ciclo de vida de los productos y lanzando familias enteras de nuevos elementos con suficientes avances técnicos y de mercadeo para inducir a los 
consumidores a adquirir nuevos productos y reemplazar los que ya tenían, incluso antes de que éstos terminen su vida útil (ZAMBON et al., 2015).

En el sector de la moda, formado por vestuario, calzado y accesorios, la mayoría de los productos presenta un ciclo de vida corto (NENNI; GIUSTINIANO; PIROLO, 2013). Por eso, las industrias competitivas realizan una sustitución constante de bienes siguiendo la dinámica del mercado, satisfaciendo así las necesidades de los consumidores.

Con el cambio de perfil de usuario, de acuerdo con Churchill y Peter (2000), el proceso de compra pasó a ser desarrollado en cinco etapas: i) reconocimiento de la necesidad; ii) búsqueda de información; iii) evaluación de alternativas; iv) decisión de compra; y v) evaluación posterior de la compra.

La evaluación post-compra, apoyada en la satisfacción del consumidor, apuntará a lo que fue preferido o descartado, revelando cuáles son los elementos elegidos por los consumidores. Bajo estas consideraciones, se deduce que la preferencia del consumidor es una función de las siguientes variables (CHURCHILL; PETER, 2000):

$$
\mathrm{P}=\mathrm{f}(M ; \mathrm{Ig} ; M k ; I m ; E)
$$

Donde:

$M=$ Memoria del consumidor potencial

$I g=$ Influencia del grupo social

$M k=$ Marketing

Im = Influencia de los medios de comunicación

$E=$ Experimentación

Las variables propuestas para la formación de las preferencias del consumidor atienden una dinámica social cuya velocidad de cambio requiere una continua reevaluación. La acción de los medios, las influencias de grupos sociales en transformación y la información diversa inducen al consumidor a una constante reconstrucción de conceptos, llevando a una memoria efímera de los productos, que son sustituidos cada vez más rápidamente.

\subsection{El Comportamiento De La Demanda De Los Productos Del Sector De La Moda}

La industria de la moda ha demostrado altos niveles de caos en el momento de prever la demanda por la cantidad de factores que influyen en sus productos (NENNI; GIUSTINIANO; PIROLO, 2013). En esta sección del artículo, analizamos ambientes principales que pueden afectar el mercado: el ambiente económico, político, social, demográfico, natural, tecnológico y competitivo. Estos aparecen en la Figura 1.

El ambiente económico es un factor clave para cualquier industria debido a la importancia de la capacidad de compra del consumidor (BESEN, 2009). Cuando los consumidores pasan por una etapa de prosperidad, el nivel de consumo sube, contrario a un estancamiento o recesión.

En el ambiente político legal, se observa cómo las leyes restringen las opciones de marketing, es decir, cuáles parámetros legales afectan las estrategias aplicadas a un producto que determinarán su precio, promoción y distribución.

El ambiente social comprende valores, creencias y comportamientos de las personas en una sociedad. Este ambiente contiene factores determinantes de las actitudes de una empresa, cuya responsabilidad social a largo plazo afecta la elección de los clientes potenciales en relación a los competidores. Esos factores son denominados psicográficos, pues identifican el estilo de vida de los consumidores, sus intereses, personalidad y opiniones. Para Cobra (1997), las personas se van volviendo progresivamente más curiosas y receptivas a la novedad. Esa característica debe nortear los análisis del mercado de la moda y sus peculiaridades.

Perspectivas em Gestão \& Conhecimento, João Pessoa, v. 7, Número Especial, p. 50-65, mar. 2017. 
Figura 1 - Ambientes que afectan el patrón de elección del consumidor de la moda

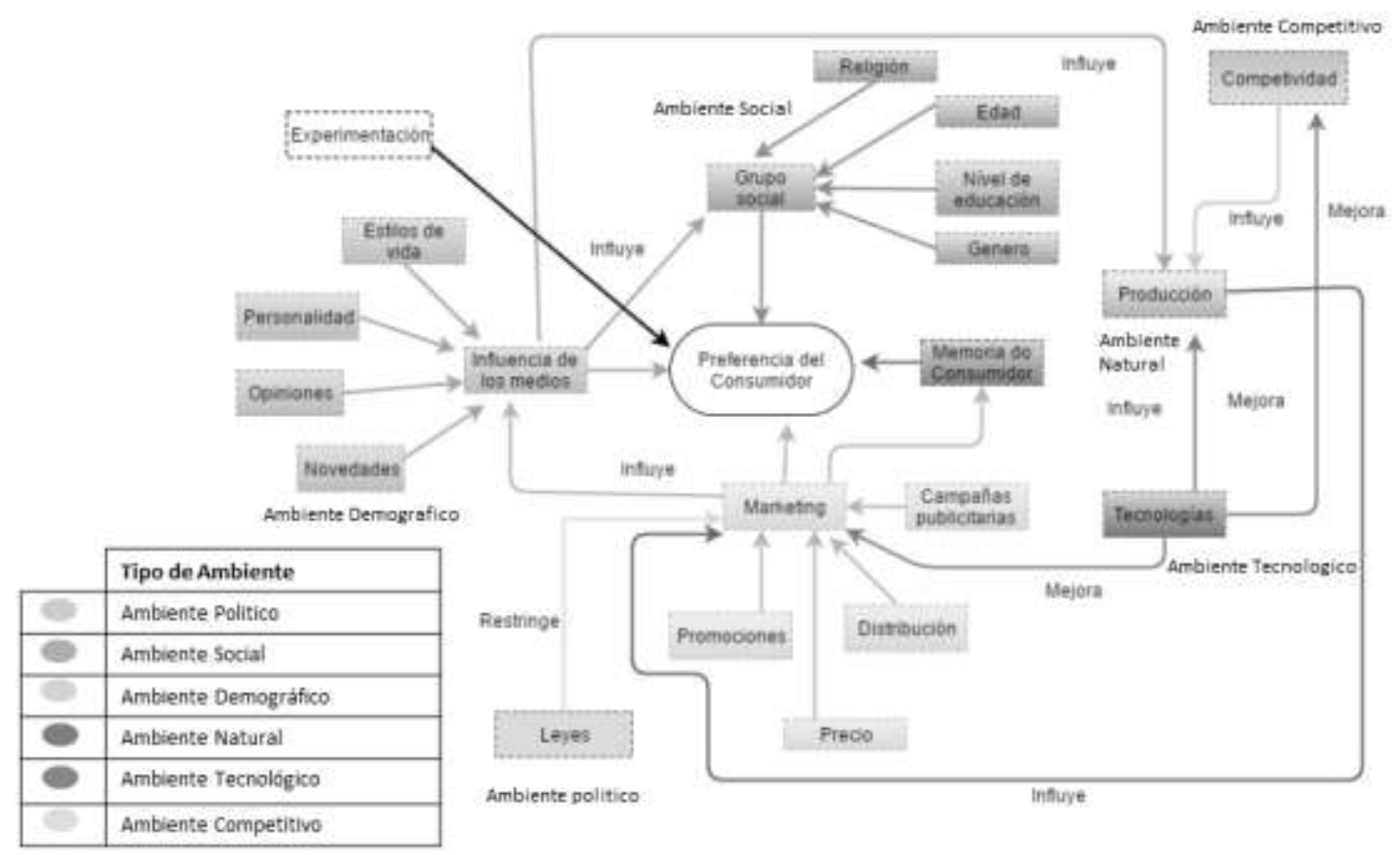

Fuente: autores

El ambiente demográfico abarca las características de una población, incluyendo el análisis de índices de natalidad, mortalidad, escolaridad y otras variables que generen cambios en la vida colectiva. Este análisis es importante para identificar y atender el mercado objetivo en relación a una acción de marketing o a la apertura de un nuevo negocio y su comportamiento. Para Cobra (1997), hacen parte de la composición de este ambiente la edad, el género, la religión, la educación y la influencia de tales elementos en la manera de vestir.

El ambiente natural revela las restricciones de explotación de los recursos que obligan a las empresas a desarrollar materiales y procesos que puedan eludir la escasez, perfeccionando tecnologías de producción para sintetizar materiales como pieles, piedras, maderas, etc. Esta condición presupone un estrechamiento de las relaciones con el ambiente tecnológico, cuyo objetivo es el desarrollo de investigaciones, invenciones e innovaciones que resulten en bienes nuevos o perfeccionados. Además de nuevos materiales, ese desarrollo tecnológico trae avances significativos para los factores de producción, para la obtención de materias primas y para la comunicación. Por ejemplo, el marketing se ve beneficiado con la mejoría en el intercambio de información, principalmente por internet, mejorando la comunicación con los clientes, convirtiéndose inclusive en una herramienta de venta.

El ambiente competitivo envuelve las actividades que ayudan a las empresas a desarrollar ventajas competitivas y a comprender el análisis de la competencia existente o potencial. La competencia es analizada identificando quiénes son los principales agentes, sus estrategias, objetivos, poder de reacción, fortalezas y debilidades.

Ante la complejidad de las variables que establecen el comportamiento de la demanda de la moda, se observa que la volatilidad en el patrón de elección ocurre en determinados segmentos de productos, separándolos en tres categorías: estilo, moda y modismo (KOTLER, 2008). Estas categorías definen frecuencias temporales distintas para sustitución de productos en el mercado. 


\subsection{El Ciclo de vida de los productos de la moda}

La frecuencia con que el mercado sustituye los productos es denominada ciclo de vida. De acuerdo con Kotler (2008), los productos de la moda se alinean a la definición clásica de producto, pero se distancian en cuanto al concepto de ciclo de vida. Para ese autor, los productos de la moda poseen tres ciclos de vida especiales, de mayor o menor volatilidad, que dependen de los cambios en las opiniones y gustos del consumidor: estilo, moda y modismo.

Los productos de ciclo de vida largo son llamados de estilo, ya que permanecen durante generaciones dentro y fuera de la moda, con varios periodos sucesivos de interés (RECH, 2007) o sea, son productos básicos (VINCENT-RICARD, 1987).

Los productos llamados de moda poseen un ciclo de vida mediano definido por una estación climática. Por ejemplo, cuál va a ser la tendencia de moda de otoño o invierno de cada año. Su curva de crecimiento es gradual cuando es aceptado - con desempeño de ventas progresivo - y su movimiento de caída es lento (RECH, 2007).

Por último, los productos llamados de modismo poseen un ciclo de vida corto. Son formados por aquellos productos que entran en el mercado rápidamente, alcanzan un auge y declinan también rápidamente. Según Kotler (2008, traducción nuestra): "el modismo dura muy poco y tiende a atraer un número limitado de seguidores".

Usualmente, los productos de la moda tienen su ciclo de vida definido en cuatro fases: análisis, elaboración, creación y difusión:

Análisis: comprende dos etapas. La primera abarca el análisis socio-cultural, es responsable por evaluar tendencias de moda, determinar el ciclo de vida del producto, la factibilidad de venta, la distribución, el descarte y la relación costo de desarrollo - retorno para la empresa. La segunda abarca el análisis socio-económico, analiza precio, segmento del mercado, público objetivo, competencia y ganancia (RECH, 2007).

Elaboración: busca poner en práctica el concepto de producto definido en la fase anterior. Hace una recolección de datos sobre nuevas tecnologías, materiales y equipos (RECH, 2007).

Creación: Para Vincent-Ricard (1987), esta fase ocurre cuando el profesional de la moda define los instrumentos utilizados para transformar el concepto de producto en algo físico, como croquis, materiales y componentes listos, llegando a una pieza piloto.

Difusión: A partir del uso de la comunicación, la publicidad y de conocimientos avanzados de marketing, esta fase es transversal a todas las anteriores, pues para ofrecer un buen producto, las empresas deben estar atentas a las necesidades de los consumidores con el fin de estipular un buen precio y definir la distribución y las promociones generadas. Según Kotler (2008, traducción nuestra):"Muchas compañías todavía planean sus productos sin pensar en las preferencias del consumidor y esos productos son rechazados en el mercado". Durante esta última fase, el producto pasa a ser evaluado con respecto a las preferencias del consumidor, definiendo si el producto será asociado a moda o modismo.

Una de las principales limitaciones para las pequeñas empresas es definir las inversiones en la producción para productos de ciclo de vida corto. Aun dentro de las condiciones mínimas de reducción del riesgo, tales esfuerzos no generan resultados significativos (NENNI; GIUSTINIANO; PIROLO, 2013). Por esta razón, es recomendable que los esfuerzos sean concentrados en la flexibilización y la racionalización del tiempo de producción, así como en la mejoría de la logística de distribución, posibilitando la reducción del tiempo entre la concepción de nuevas piezas, su producción y entrega. Sin embargo, se observa que las estrategias de distribución más eficaces no son adoptadas por las pequeñas empresas, pero sí por las grandes redes de tiendas minoristas denominadas fast fashions. 


\subsection{Conceptos Fast Fashion y atendimiento de los patrones contemporáneos de demanda}

El concepto de tienda fast fashion (moda rápida) surgió a partir de iniciativas de grandes redes minoristas. Este concepto surgió en la red de tiendas Zara, que actúa en el mercado de vestuario femenino, masculino e infantil (DELGADO, 2008; SULL; TURCONI, 2008). El fast fashion resultó eficiente en el aumento de la facturación a partir del final de la década de 1980, proporcionando a Zara un amplio reconocimiento en el mercado global.

El concepto de fast fashion es también denominado como "supermercado de estilos" y parte del principio de la diversificación de la oferta, que atiende a la actual dinámica del ciclo de vida de moda y modismo en los productos. Con esto se busca garantizar la libertad del consumidor de entrar a un establecimiento y escoger los productos de acuerdo con su estilo (POLHEMUS, 1994). Entretanto, la propuesta real de los adeptos a este concepto es intentar absorber con rapidez las informaciones del ambiente social y demográfico, atendiendo la demanda de productos con ciclo de vida corto (SAPPER, 2010).

Gracias a la gran variedad de artículos ofrecidos por las tiendas fast fashion en un ámbito global, la variedad de modelos de los productos expuestos, asociada a pequeñas cantidades, induce la sensación de semi-exclusividad, o de una alta tasa de sustitución (ERNER, 2005).

Otra concepción que acentúa la preferencia del consumidor por las fast fashion es que este tipo de establecimiento posee ropas y accesorios básicos, o sea, pertenecientes al concepto de estilo, y principalmente productos representantes del modismo, que divulgan las nuevas tendencias del sector. Esta característica marcante de las fast fashion permite una amplia investigación de consumo, puesto que puede observarse qué están consumiendo realmente las personas en términos de moda, para luego iniciar el proceso de creación y producción de los productos. De esta manera, según Erner (2005, traducción nuestra): "el concepto fast fashion...privilegia las tendencias en detrimento de la creatividad".

En resumen, entre las principales características de esta estrategia están: la rotación de las colecciones, las variedades de prendas y la rapidez con que estas son sustituidas debido, en gran parte, a la previsión temprana de la demanda. La capacidad de predecir adecuadamente la demanda es particularmente importante para empresas como Zara, porque una de sus estrategias fundamentales es mantener bajos inventarios en los almacenes y reponerlos dos veces por semana sobre la base de las expectativas de demanda (GARCIA, 2014). Por eso tienen la capacidad de adaptar su oferta rápidamente a las nuevas necesidades y gustos de los consumidores, anticipándose a su competencia. La estrategia fast fashion, favorable a las grandes redes minoristas, nos lleva a preguntarnos si ella podría ser usada en pequeñas y medianas industrias aumentado su competitividad.

\section{METODOLOGÍA: HERRAMIENTAS DE GESTIÓN DEL CONOCIMIENTO UTILIZADAS}

La gestión del conocimiento es un proceso enfocado en la estrategia colectiva que envuelve la gestión de competencias y de capital intelectual, el aprendizaje organizacional, la inteligencia empresarial y la educación corporativa (NONAKA; TAKEUCHI; UMEMOTO, 1996). La gestión del conocimiento en las empresas debe orientarse a la formación de un círculo virtuoso de conversión de conocimiento tácito en conocimiento explícito y viceversa (LIEBOWITZ; CHEN, 2001). De esta manera, la gestión del conocimiento se torna un proceso central en la búsqueda y obtención de inteligencia competitiva, en virtud de ampliar el acceso a la información. Entretanto, solo el acceso a la información no amplía el nivel de conocimiento corporativo, ya que este último también depende de un abordaje sistémico para relacionar las informaciones entre sí, transformándolas en conocimiento. Los modelos de análisis usados en abordajes sistémicos se encuentran ampliamente descritos en la literatura actual. Estos se apoyan frecuentemente en mapas conceptuales y simulaciones. 


\subsection{Mapas conceptuales y representación del conocimiento}

Las empresas son ambientes de trabajo colectivo y buscan construir conocimiento en grupo. Para externalizar ese conocimiento, las empresas deben promover la socialización y el compartimiento del conocimiento entre sus colaboradores (NONAKA; TAKEUCHI; UMEMOTO, 1996; SENGE, 1997). Una condición para que el conocimiento sea compartido entre los diversos agentes es su representación. Para representar conocimiento eficientemente, es necesario un método formal y sistemático que explicite el conocimiento tácito relevante que cada individuo posee, construyendo inteligencia organizacional (ZAMBON et al., 2016a). El software BLUE KMS ${ }^{\circledR}$ (ZAMBON et al., 2016b) usa un modelo de representación del conocimiento apoyado en mapas conceptuales para ambientes de gestión estratégica.

Los mapas conceptuales son diagramas que relacionan conceptos, es decir, relacionan pequeñas frases que contienen un sustantivo, el cual es el centro del significado. Los conceptos tienen relaciones de causa y efecto entre sí que son representadas por verbos (NOVAK, 2010). Dos conceptos interligados por un verbo originan una proposición. En BLUE KMS ${ }^{\circledR}$, una proposición inicial $(P)$ introduce un problema o un tema y otras proposiciones $(p)$, que se relacionan con $P$, amplían su interpretación, agregando nuevos conceptos subsidiarios y ampliando su comprensión. Los conceptos son posicionados en una matriz de atributos que representa las influencias externas que modifican las condiciones originales de los componentes internos del problema.

Una matriz de atributos es un instrumento analítico que permite categorizar conceptos por medio de criterios, que a su vez, representan segmentos de diferenciación, como se muestra en la Figura 2. En ella, aparecen conceptos que explican o extienden el conocimiento sobre P, presentada sobre la matriz de atributos. Los conceptos se posicionan en la matriz en términos de controlabilidad sobre el escenario de análisis (columnas) y en términos de controlabilidad del agente de decisión (líneas). En ambos ejes, cada concepto se asocia con el problema que está siendo analizado en tres segmentos que vinculan cada concepto a $\mathrm{P}$ con una escala de fuerzas que se va atenuando de derecha a izquierda y de arriba hacia abajo en el siguiente orden: Controlable, Penumbra y No Controlable (CT, PN, NC).

Figura 2 - Modelo de representación del conocimiento según BLUE KMS ${ }^{\circledR}$

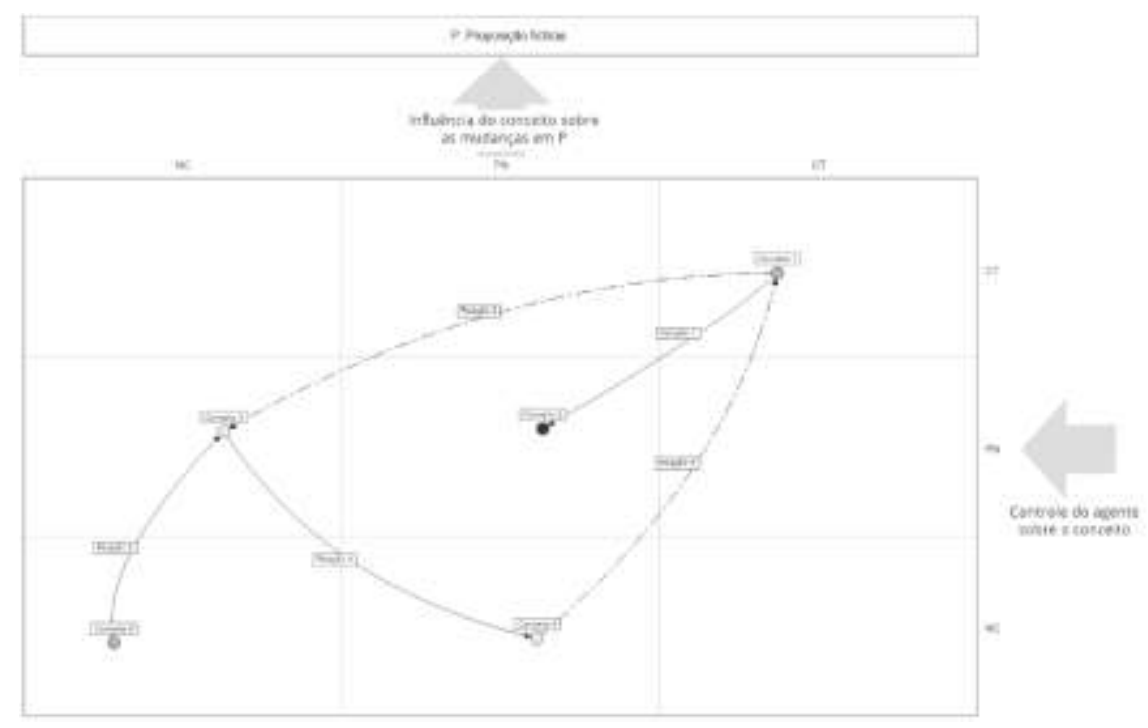

Fuente: autores 
Según lo anterior, la relación del agente del que se extrae el conocimiento con $P$ se encuentra en las líneas de la matriz y en su capacidad de hacer cambios en el estado de los conceptos extendidos de $P$. La relación de cada concepto contenido en la matriz con $P$ se encuentra en las columnas y refleja la capacidad de dicho concepto de hacer cambios en el estado de $P$. Los segmentos clasificados como $\mathrm{CT}$, poseen atributos de dependencia para la realización de cambios en el sistema. Los segmentos NC, poseen atributos de autonomía respecto a los cambios que imponen en el sistema. Por último, los segmentos PN, reflejan la incerteza del sistema (ZAMBON et al., 2016a).

La relación entre los conceptos y la intensidad de esa relación aparece en términos de relaciones causales, donde la transferencia de energía de un concepto sobre otro es dada por un verbo (ZAMBON et al., 2016a), vigorizado por un vínculo de refuerzo o balanceo (SENGE, 1997). Un balanceo (línea guion-punto) ocurre cuando hay una diferencia entre el estado actual y el estado deseado de una variable, generando acciones que buscan eliminar esa diferencia. Un refuerzo (línea continua) representa un mecanismo de crecimiento en el sistema. El estado del sistema, que se desarrolla de manera continua como un efecto de bola de nieve, es representado por una tasa de cambio.

\subsection{Simulación por medio de dinámica de sistemas}

Los postulados de la DS (FORRESTER, 1958) están asociados a la teoría de realimentación de información y control como medio de análisis de sistemas humanos y naturales. Esta teoría considera todo sistema como complejo y sus componentes son explicados como estoques y flujos. Los segundos asocian a los primeros en causa y efecto. Al conectarse, los estoques forman ciclos, posibilitando el análisis de las realimentaciones responsables de la complejidad del sistema (ZAMBON, 2006).

A partir de una visión general, la DS asume que el análisis de una situación puede ser realizada desde un punto de vista objetivo externo y que la estructura y los procesos dinámicos del mundo real pueden ser analizados en diagramas con simbología específica y modelos matemáticos. En un contexto organizacional, las situaciones pueden ser caracterizadas por la complejidad de la estructura de elementos y círculos de causalidad existentes entre esos elementos. La información generada por un elemento introduce cambios en la información generada por otros elementos y así sucesivamente, ocasionando una compleja red de cambio de informaciones, que analizadas conjuntamente pueden explicar el fenómeno de cambio (FLOOD, 1998). El modelaje y simulación de un sistema comprende relaciones, interacciones y atrasos, estimulando la comprensión del sistema real holística y anticipativamente. Por lo tanto, los resultados obtenidos pueden subsidiar resoluciones en campos en donde apenas cabía la intuición (THOMPSON; BANK, 2010).

Partiendo de la premisa de que es posible modelar un sistema complejo, reconocer los círculos de causalidad, las perturbaciones y ruidos que el modelo pueda presentar, se pueden recrear situaciones a partir de la situación real, probando ideas o creando controles más eficaces. Los resultados deseables pueden implementarse en la realidad si las acciones implementadas en la simulación que hayan convergido para resultados indeseables puedan suprimirse.

\section{RESULTADOS: MODELADO Y SIMULACIÓN DEL MODELO DE PREFERENCIA DEL CONSUMIDOR}

Según la función propuesta por Churchill y Peter (2000) para deducir la preferencia de consumidor y teniendo en cuenta los ambientes descritos en la sección tres de este artículo, se creó un modelo que describe las diferentes variables que de una u otra forma pueden afectar la preferencia del consumidor y cómo ellas se relacionan entre sí.

Perspectivas em Gestão \& Conhecimento, João Pessoa, v. 7, Número Especial, p. 50-65, mar. 2017. 


\subsection{Modelado y simulación del modelo de preferencia}

Para analizar el problema sistémicamente, fue empleado el software BLUE KMS ${ }^{\circledR}$ (ZAMBON et al., 2016b), obteniendo el MCE que sirvió para realizar el análisis sistémico del modelo de preferencia del consumidor descrito en la Figura 1 (CHURCHILL; PETER, 2000). El MCE resultante se muestra en la Figura 3.

Figura 3 - MCE del modelo de preferencia del consumidor

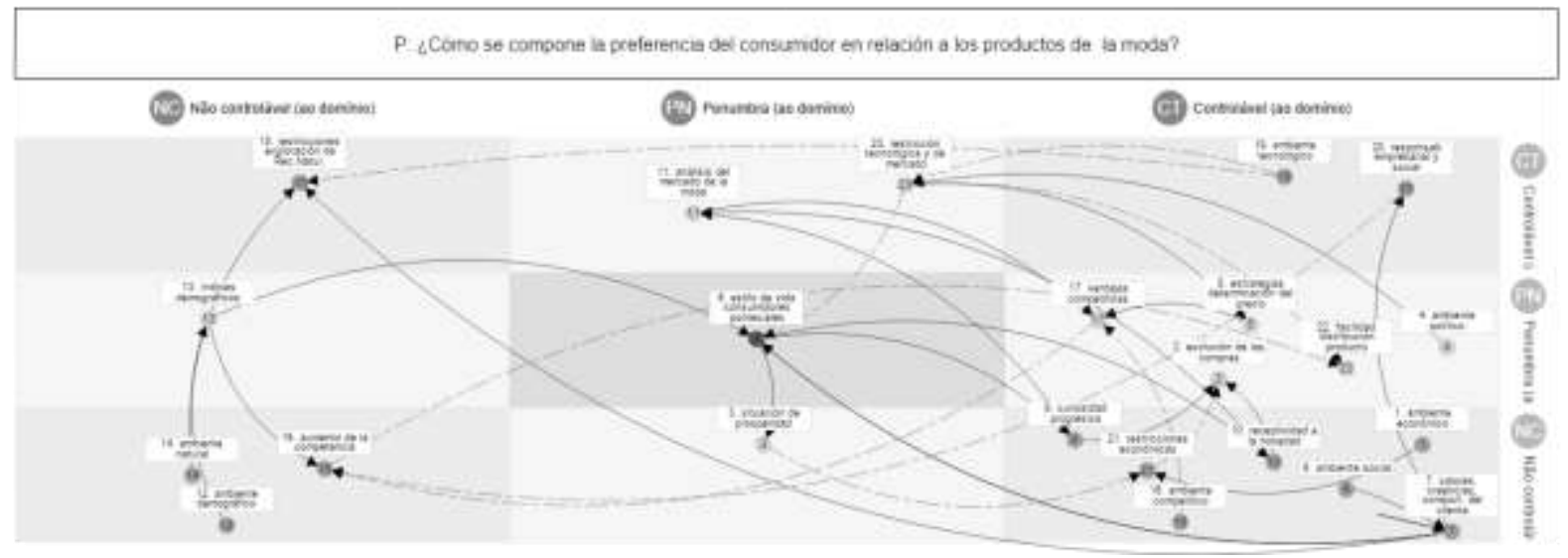

Fuente: autores

Siguiendo el MCE de la Figura 3, se observa que el área central comprende un ambiente de incerteza. En ella se encuentra un punto importante en el análisis sistémico de la preferencia del consumidor, ya que está relacionado con otras seis variables: "estilo de vida de los consumidores potenciales" (8).

El estilo de vida resulta de una sobreposición de los ambientes natural, demográfico, social, tecnológico y económico, siendo imposible identificar los niveles de impacto que cada ambiente ejerce sobre él, en virtud de la complejidad de las relaciones. Analizando las relaciones directas, el estilo de vida refuerza la "receptividad a la novedad" (10) y la "curiosidad progresiva" (9) sobre la "evolución de las compras" (2).

Acertadamente, ambos conceptos (9 y 10) son considerados en "el análisis del mercado de la moda" (11). No obstante, el análisis del mercado es realizado con base en conceptos existentes en el ámbito no controlable (Não Controlável - NC) por los agentes y, no necesariamente, surte efecto sobre la identificación del patrón de preferencia del consumidor.

Como agravante, el análisis del mercado influencia todo el ambiente competitivo, buscando definir "ventajas competitivas" (17) que están asociadas a las "estrategias de determinación de precios" (5). Tales estrategias están directamente relacionadas al "ambiente tecnológico" (19), que impone "restricciones tecnológicas y de mercado" (23) y presiones al "estilo de vida de los consumidores potenciales" (8). Así, se genera un círculo vicioso que impone un "aumento de la competencia" (18), creando barreras a la "facilidad de distribución de los productos" (22) en razón de la ampliación de la oferta de novedades.

Estas condiciones fueron identificadas sobretodo en ambientes donde coexisten pequeñas empresas con baja capacidad de manipular adecuadamente las variables referentes al desarrollo tecnológico y a la evaluación de los conceptos de novedad e innovación. Para atender esta dificultad, proponemos una estrategia basada en el concepto de fast fashion que busca minimizar los problemas que puedan presentarse. 


\subsection{Adaptación del modelo fast fashion en las pyme}

Como se mencionó, el modelo fast fashion está diseñado especialmente para atender la dinámica en los productos de moda y modismo. Dos características principales son producir pequeñas cantidades de cada producto, aumentado la sensación de exclusividad y disminuir el tiempo entre el diseño y la producción, respondiendo rápidamente a cambios de preferencia.

Esta estrategia representa inicialmente un incremento significativo en los costos y podría verse como un método no accesible para pequeñas y medianas empresas. No obstante, los beneficios de adaptar la oferta a la demanda actual usualmente sobrepasan los costos de producción, ayudando a reducir los casos en que grandes volúmenes de inventario deben venderse con grandes descuentos o en el peor caso la perdida de los productos (GARCIA, 2014). Por ejemplo, Ghemawat y Nueno (2006) demostraron que los minoristas de moda como Zara podrían perder entre el 15 y el $20 \%$ de las ventas provenientes de artículos con descuento, debido a que manejan pocas cantidades por producto, mientras que los minoristas tradicionales tienen pérdidas en el rango de $30 \%-40 \%$.

El fast fashion no busca producir diversas variedades de productos aleatoriamente sino, por el contrario, producir los productos necesarios para satisfacer a los consumidores. La productividad mostrada influye altamente en la sensación de exclusividad del consumidor, la cual depende de diversos factores. Con el objetivo de conocer al consumidor y los productos que él busca, empresas como Zara han invertido en el uso de tecnologías de información y comunicación. Estas tecnologías usan la información recogida en las tiendas a la hora de la compra de los clientes, datos históricos, y otras informaciones, desarrollando un co-aprendizaje (GARCIA, 2014) que les garantiza un mayor acierto en su producción, adaptando su oferta a las demandas actuales. El desconocimiento sobre los intereses del consumidor y el alto costo de inversión en tecnologías muchas veces se convierten en una desventaja para las PYME (ZAMBON; ANUNCIAÇÃO, 2014), impidiendo su adaptación al fast fashion.

Con base en los aspectos mencionados a lo largo de este artículo que describen características claves del modelo fast fashion y con el fin de sintetizar los resultados encontrados en la revisión de la literatura, presentamos un diagrama causal. A partir de este concepto se creó una simulación en DS usando el software STELLA 9.1.3, del modelo ideal de fast fashion descrito en la Sección 3.3. Los resultados son mostrados en la Figura 4.

Figura 4 - a. Modelo DS de la estrategia fast fashion. b. Relación entre las curvas de costos de producción y ganancia
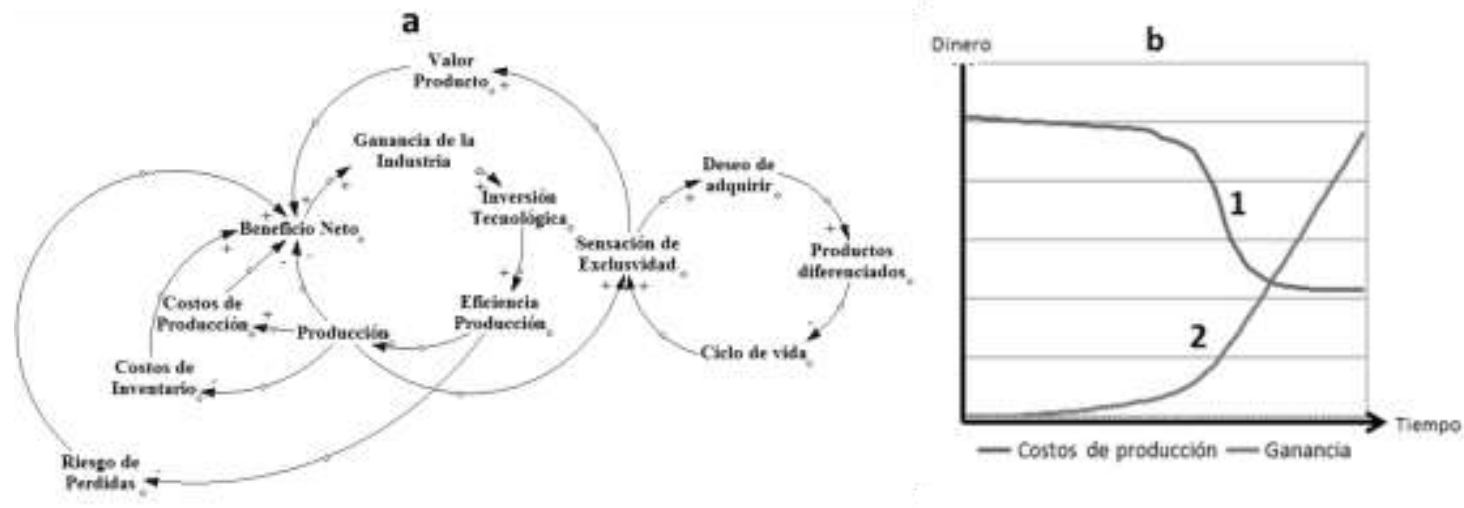

Fuente: autores

Según lo mostrado en la Figura 4a, la estrategia de producción de las PYME debe basarse en la eficiencia en la producción y la en la inteligencia del mercado, a través del conocimiento sobre las

Perspectivas em Gestão \& Conhecimento, João Pessoa, v. 7, Número Especial, p. 50-65, mar. 2017. 
preferencias del consumidor, creando productos con el mínimo de recursos (eficiencia en producción) y ajustados a la demanda (inteligencia del mercado).

El diagrama de la Figura 4a muestra cómo un aumento en la inversión en tecnología mejora la eficiencia de la producción (+), llevando a una disminución en la producción de un producto específico (-), ya que solo se produce lo requerido por el mercado. Esta situación aumenta los costos de producción. Sin embargo, este aumento es compensado con la reducción de costos de inventario, un menor riesgo de pérdida y el aumento del valor del producto, obteniendo una mayor ganancia para la industria. Por otro lado, una producción en pequeños lotes crea una sensación de exclusividad, aumentando el deseo de adquirir por parte de los consumidores.

Así mismo, esta estrategia produce una mayor diversidad de productos y disminuye el ciclo de vida de cada producto, generando una sensación de exclusividad en el cliente. La Figura $4 \mathrm{~b}$ compara el comportamiento entre los costos de producción (Curva 1) y la ganancia generada (Curva 2) en la estrategia fast fashion. Inicialmente, los altos costos de producción reducen la ganancia. Sin embargo, a medida que la inversión aumenta y mejora la previsión de la demanda, las ganancias son mayores. Estos resultados reflejan que conocer al consumidor y producir en torno a sus deseos se genera una mayor ventaja competitiva al alcance de las PYME.

\section{CONCLUSIONES}

En este artículo, abordamos el problema de las pequeñas y medianas empresas para decidir qué, cuánto y cómo producir en un ambiente complejo, partiendo del modelo de preferencia del consumidor. Dicho modelo es un punto fundamental a tener en cuenta en la cadena de producción, debido a su influencia sobre las ganancias de la empresa. Un ejemplo de estrategias que han tenido éxito y están basadas en los nuevos gustos e intereses del consumidor, es la estrategia redes fast fashion, la cual establece relaciones de preferencia (ELLSBERG, 1954) de sus clientes potenciales, obteniendo ventajas competitivas claras (NENNI; GIUSTINIANO; PIROLO, 2013). Sin embargo, definir cuáles son las preferencias del consumidor en determinado momento y trabajar en torno a ellas, no es una tarea fácil y requiere de diferentes tecnologías que permitan extraer conocimiento sobre los consumidores.

Este trabajo permitió comprender, a través del modelo de preferencia del consumidor (CHURCHILL; PETER, 2000) y la identificación de los ambientes influyentes del mercado, la complejidad del patrón de preferencia del consumidor, el cual es un proceso dependiente de diversas variables, interrelacionadas. También, por medio del modelo representativo de la estrategia fast fashion simulado en el software STELLA 9.1.3 (ISEESYSTEMS, 2009), fue posible identificar la relación entre los costos de producción y las ganancias considerando la relación entre las diferentes variables que afectan la preferencia del consumidor.

Los estudios realizados permitieron caracterizar el modelo propuesto por Churchill y Peter (2000), el cual es un modelo conceptual. Dicho modelo debe ser testado en la práctica, buscando alternativas para poder encontrar información relevante y actual sobre el consumidor de la moda. También, a la hora de aplicar un modelo en un ambiente productivo, deben considerarse la realidad social y la diversidad de opiniones presentes en el mercado estudiado.

Contando con datos reales y con un modelo más ajustado, los instrumentos de gestión de conocimiento pueden ayudar a las empresas a aumentar su competitividad al apuntar las inversiones a productos atractivos para el consumidor. Otros trabajos futuros deberán enfocarse en nuevos métodos de análisis de patrones de elección del consumidor, específicamente en métodos de gestión de conocimiento aplicados, que puedan producir mejores estrategias para alcanzar la dinámica necesaria en la cadena de la moda.

Perspectivas em Gestão \& Conhecimento, João Pessoa, v. 7, Número Especial, p. 50-65, mar. 2017. 


\section{REFERENCIAS}

AGIS, D. et al. Vestindo o futuro: microtendências para as indústrias têxtil, vestuário e moda até 2020. 1a. ed. Vila Nova de Famalicão: ATP, 2010.

ALVEREZ, F. J. S. M.; FAVERO, M. B.; FRANCISCO, G. D. Marcas de Moda e a Percepção Dos Consumidores. Projética, v. 4, p. 65-82, 2013.

BESEN, F. Marketing e portfólio de moda. Disponível em: http://docplayer.com.br/2169897Marketing-e-portfolio-de-moda-professora-fabiana-besen-2009-1-1.html. Acesso em: 15 fev. 2016.

CHEGINI, F.; MOLAN, S. B.; KASHANIFAR, S. S. An Examination of the Impact of Cultural Values on Brand Preferences in Tehran's Fashion Market. Procedia Economics and Finance, v. 36, n. 16, p. 189200, 2016.

CHURCHILL, G. A.; PETER, P. J. Marketing: criando valor para os clientes. 2. ed. São Paulo: Saraiva, 2000.

COBRA, M. Algumas reflexoes acerca do marketing da moda. RAE Light, v. 4, n. 4, p. 2-5, 1997.

DELGADO, D. Fast fashion: estratégia para conquista do mercado globalizado. Modapalavra, v. 1, n. 3, p. 3-10, 2008.

ELLSBERG, D. Classic and Current Notions of "Measurable Utility". The Economic Journal, v. 64, n. 255, p. 528-556, 1954.

ERNER, G. Vítimas da moda? como a criamos, por que a seguimos. São Paulo: Senac, 2005.

FLOOD, R. "Fifth Discipline": Review and Discussion. Systemic Practice and Action Research, v. 11, n. 3, p. 259-273, 1998.

FORRESTER, J. W. Industrial Dynamics. Harvard Business Review, v. 36, n. 4, p. 37-66, 1958.

GARCIA, J. M. Demand Forecasting at Zara: A Look at Seasonality, Product Lifecycle and Cannibalization. 2014.

GHEMAWAT, P.; NUENO, J. L. ZARA: Fast Fashion. Harvard Business School, n. October 2015, 2006.

HALUK KÖKSAL, M. Consumer behaviour and preferences regarding children's clothing in Turkey. Journal of Fashion Marketing and Management: An International Journal, v. 11, n. 1, p. 69-81, 6 mar. 2007.

ISEESYSTEMS. STELLA. Disponível em: http://www.iseesystems.com.

ISMAIL, Z.; MASOOD, S.; TAWAB, Z. M. Factors Affecting Consumer Preference of International Brands over Local Brands. In: INTERNATIONAL CONFERENCE ON SOCIAL SCIENCE AND HUMANITY, 2nd., Singapore, v. 31, p. 54-59, 2012.

KARIMI, S.; PAPAMICHAIL, K. N.; HOLLAND, C. P. The effect of prior knowledge and decision-making style on the online purchase decision-making process: a typology of consumer shopping behaviour. Decision Support Systems, v. 77, p. 137-147, set. 2015.

KOTLER, P. Princípios de marketing. 12. ed. Rio de Janeiro: Prentice Hall, 2008.

LIEBOWITZ, J.; CHEN, Y. Developing Knowledge-Sharing Proficiencies. Knowledge Management Review, v. 3, p. 12-15, 2001.

MDIC. Sistema Moda Brasil. Disponível em: http://www.sistemamodabrasil.com.br/. Acesso em: 1 maio. 2015.

NENNI, M. E.; GIUSTINIANO, L.; PIROLO, L. Demand forecasting in the fashion industry: a review. 
International Journal of Engineering Business Management, v. 5, n. SPL.ISSUE, 2013.

NONAKA, I.; TAKEUCHI, H.; UMEMOTO, K. A theory of organizational knowledge creation. International Journal of Technology Management, v. 11, n. 7-8, p. 833-845, 1996.

NOVAK, J. D. Learning, creating, and using knowledge: concept maps as facilitative tools in schools and corporations. 2th. ed. New York: [s.n.]. v. 6.

PORTER, M. E. Estratégia competitiva: técnicas para análise de indústrias e da concorrência. 3. ed. Rio de Janeiro: Campus, 1986.

PRICEWATERHOUSECOOPERS. As pequenas e médias empresas no Brasil. [s.l: s.n.]. Disponível em: https://www.pwc.com.br/pt/publicacoes/setores-atividade/assets/pcs/private-compay-services-pcs13-pt.pdf.Acceso em: 15 fev. 2016.

RECH, S. R. Conceitos de produto de moda. Disponível em: http://fido.palermo.edu/servicios dyc/encuentro2007/02 auspicios publicaciones/actas diseno/art iculos pdf/A7012.pdf. Acceso em: 15 fev. 2016.

SAPPER, S. L. Consumo: a engrenagem do fast fashion. DAPesquisa, v. 8, 2010.

SENGE, P. M. The fifth discipline measuring business excellence, 1997.

SULL, D.; TURCONI, S. Fast fashion lessons. Business Strategy Review, v. 19, n. 2, p. 4-11, 2008.

THOMPSON, B. P.; BANK, L. C. Use of system dynamics as a decision-making tool in building design and operation. Building and Environment, v. 45, n. 4, p. 1006-1015, 2010.

UNIETHOS. Sustentabilidade e competitividade na cadeia da moda. São Paulo: [s.n.]. Disponível em: http://www.abit.org.br/conteudo/links/estudo sustentabilidade uniethos.pdf. Acceso em: $15 \mathrm{fev}$. 2016.

VINCENT-RICARD, F. As espirais da moda. [s.I.] Paz e Terra, 1987.

WOMACK, J. P.; JONES, D. T.; ROOS, D. The machine that changed the world: The story of lean production:Toyota's secret weapon in the global car wars that is now revolutionizing world industry. [s.l.] Simon and Schuster, 2007.

ZAMBON, A.; ANUNCIAÇÃO, P. Inteligência Competitiva Percepções de valor no setor da bijuteria. Revista Portuguesa e Brasileira de Gestão, p. 1-20, 2014.

ZAMBON, A. C. Uma contribuição ao processo de aquisição e sistematização do conhecimento multiespecialista e sua modelagem baseada na Dinâmica de Sistemas. Tese (Doutorado) Universidade Federal de São Carlos, 2006.

ZAMBON, A. C. et al. Obsolescência acelerada de produtos tecnológicos e os impactos na sustentabilidade da produção. Revista de Administração Mackenzie, v. 16, n. 4, p. 231-258, 2015.

ZAMBON, A. C. et al. Uma aplicação prática de gestão do conhecimento e simulação na resolução de problemas complexos empresariais. Revista Produção Online, v. 16, n. 2, p. 408-440, 2016 a.

ZAMBON, A. C. et al. BLUE KMS. Limeira, SP - BrazilINPI - Proc. BR 512016000860 1, 2016 b.

Artigo recebido em 06/07/2016 e aceito para publicação em 23/02/2017 